\title{
A New Combined Strategy for Discrimination between Types of Weed
}

\author{
P. Javier Herrera ${ }^{1}$, José Dorado ${ }^{2}$, and Ángela Ribeiro ${ }^{1}$ \\ ${ }^{1}$ Centre for Automation and Robotics, CSIC-UPM, 28500 Madrid, Spain \\ ${ }^{2}$ Institute of Agricultural Sciences, CSIC, 28006 Madrid, Spain \\ \{pj.herrera, angela.ribeiro\}@csic.es, jose.dorado@ica.csic.es
}

\begin{abstract}
Specific weed management consists on adjusting herbicide treatments depending on the zone infested and the type of weed. In this context, the discrimination between grasses (monocots) and broad-leaved weeds (dicots) is an important objective mainly because the two weed groups can be appropriately controlled by different specific herbicides. This work proposes a method of discrimination between these types of weeds based on a combined strategy, the Sugeno Fuzzy Integral, where the final decision is taken by combining seven attributes, the Hu moments. The main challenge in terms of image analysis is to achieve an appropriate discrimination between both groups in outdoor field images under varying conditions of lighting as well as of soil background texture.
\end{abstract}

Keywords: Precision Agriculture, weed discrimination, monocots/dicots discrimination, Sugeno Fuzzy Integral, colour segmentation, Hu moments.

\section{Introduction}

Precision Agriculture is intended to adjust the use of resources and agronomic practices to the requirements of soil and crop seeking greater sustainability and efficiency. In other words, aims to reduce economic and environmental costs traditionally associated with agriculture.

The application of herbicides for agricultural purposes has been realised for decades with relevant gains in crop production. However, excessive or careless use of certain herbicides can have detrimental effects on the surface water and ground water quality. There is no doubt that the use of selective herbicide application on weedinfested areas of the field, rather than the entire field, would be of great interest [1]. As a result, efforts are being encouraged by concern over reducing agricultural chemical use without sacrificing crop yield.

Several authors show that the distribution of the most harmful weeds for a particular crop is not uniform, and it generally (about a $70 \%$ of the fields) affects less than $40 \%$ of the crop [2,3]. Due to this fact, the selective application of herbicides only on infested areas can lead to significant reductions in the amount of product applied and provide both economic and ecological benefits [4 - 6]. From the point of 
view of herbicide treatment, efficiency is higher if selective treatment is performed for each type of weed instead of using a wide spectrum herbicide [7].

To adapt the treatment to the individual needs of each kind of land, it is essential to have accurate information on the state of the crop to be treated, i.e. where the weeds are located, the degree of coverage or type of infestation present. Traditionally, different methods have been used to obtain information of the field from the air and the ground. In the case of aerial images and data coming from satellites, information collection depends heavily on the weather (no clouds or fog) and, although remote sensing in agriculture has experienced a resurgence in recent years thanks to the use of cameras hyper and multi-spectral [8], yet the cost is high and the resolution is low. Ground sampling allows a lower resolution (centimetre) although in this case the information contained in each image covers small crop areas.

The development of methods of detection of weeds from images has always been an open field of great importance to Precision Agriculture [9 - 13]. The problem has no simple solution owing to the great diversity of crops and weeds, changes in exterior lighting, differences in the texture of the terrain (fundamentally due to humidity), different states of crop growth and infestation, great similarities between the crop and weeds that infest it, etc. [14, 15]. All this makes the discrimination between the crop, weed and soil a complex task, and the difficulty increases if the aim is to discriminate between groups of weeds or to apply herbicide in real time as the position of the infestation is detected [4, 16 - 20].

One of the key criteria for herbicide selectivity is based on differences between monocots and dicots. For this reason, the determination of the coverage percentages of both groups is essential to the development of an autonomous system of treatment able to adjust the type of herbicide and the dose to the dominant infestation.

The literature contains several examples of works that propose methods of image processing that combine colour, position, outline, texture, size, or spectrum to distinguish between weeds and crops [21, 22]. Other works present combinations of image processing techniques as Fuzzy Clustering and a fuzzy inference neural network to identify plants, based on leaves [23].

The success with which these aspects can be adapted to classification depends on the kind of crop, weeds, manner and moment that the images are gathered. In other words, early weed detection is an objective that can be planned according to criteria oriented to two different levels with an increasing requirement: 1) estimation of presence or absence of weeds by discrimination from bare soil and row crops, and 2) differentiation between groups of weeds e.g., monocots vs. dicots (since they can be controlled by different herbicides) according to one or several differential parameters (spectral characteristics, and other features as size or form). Previous works have faced this problem by means of techniques as neural networks or genetic algorithms $[21,24]$.

Changing the subject, moment invariants were firstly introduced to the pattern recognition community in 1962 by $\mathrm{Hu}$ [25], who employed the results of the theory of algebraic invariants and derived his seven famous invariants to rotation of 2-D objects. Since that time, numerous works have been devoted to various improvements and generalizations of Hu's invariants and also to its use in many application areas. 
Invariance with respect to translation, rotation and scaling is required in almost all practical applications, because the object should be correctly recognized, regardless of its position and orientation in the scene and of the object-to-camera distance [26, 27].

Weed spatial distributions are unique, with monocot infestations more patchy than dicots [3]. Besides, monocots differ architecturally from dicots as one can see in Fig. 1. For these reasons, a strategy based on using Hu moments in shape recognition may be suitable. Moreover, these moments have been used successfully in previous works solving different problems but where images present similar features (outdoor images, changes in exterior lighting, varying conditions of lighting, differences in the textures, overlapping, etc) [28].

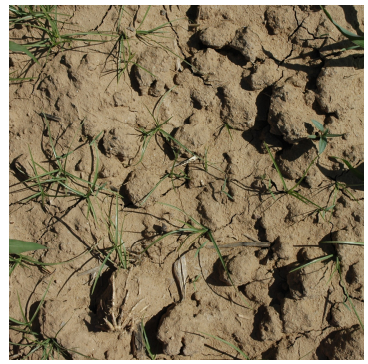

(a)

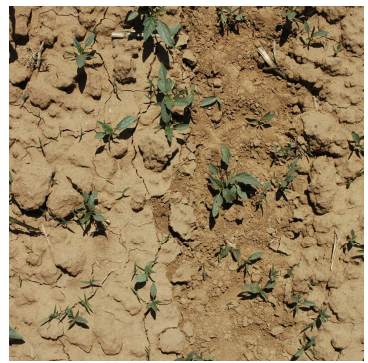

(c)

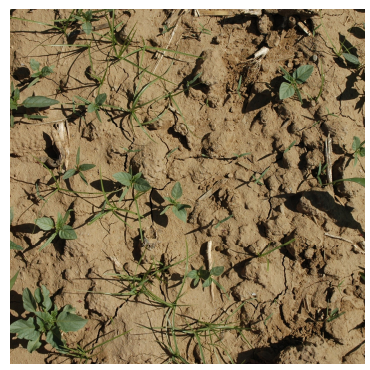

(e)

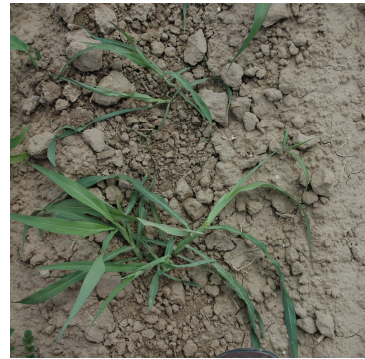

(b)

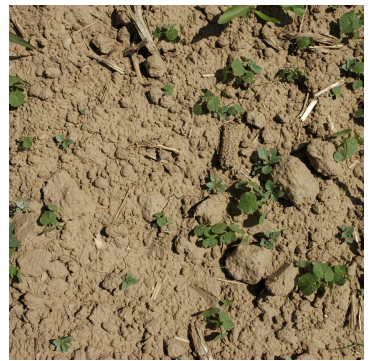

(d)

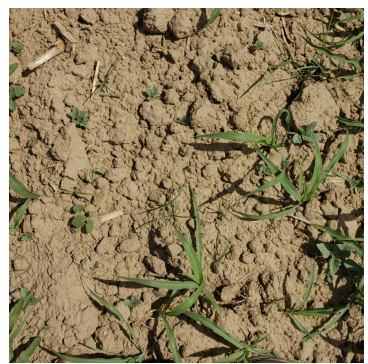

(f)

Fig. 1. Images (a) and (b) show monocots (long and slender leaf), whereas images (c) and (d) present dicots (broadleaf and short). Images (e) and (f) display a mixture of both kinds of weeds. 
This work constitutes a new approach to discrimination among groups of weeds. In particular, this work presents a novel method of discrimination between monocots and dicots, based on the support that each region belonging to weed receives by combining attributes. Our hypothesis is that a region belonging to a weed species can be characterized by a set of seven attributes based on the seven invariant moments of Hu. Furthermore, the Sugeno Fuzzy Integral (SFI) approach is used for the combination, so the decision about the kind of weed for a region, can be made according to the support that each region receives by combining the attributes by means of the SFI.

SFI has been reported to give excellent results as a classifier combiner [29]. Moreover, based on the conclusions reported in [28, 30 - 32], the SFI appears as a suitable method for the combination of attributes. In fact, with a little adjusting it can be used for combining attributes in this proposal, so that a decision about a unique kind of weed (monocot or dicot) can be made for each region.

Summarizing, the combined SFI strategy makes possible an automatic way to distinguish between different kinds of weed in outdoors images. The final purpose is to estimate the coverage percentages of each type of weed in the image.

This work is organized as follows. Section 2 describes the proposed approach, including a brief overview of the SFI. Section 3 describes the results obtained by using the combined approach. Section 4 presents the conclusions and future work.

\section{Proposed Approach}

An essential issue in the field of pattern analysis is the recognition of objects and characteristics of these objects regardless of their position, size and orientation. The idea of using moments in shape recognition gained relevance when $\mathrm{Hu}$ [25], derived a set of invariants using algebraic invariants. In particular, Hu defined seven values, computed by normalizing central moments through order three that were invariant to object scale, position, and orientation.

Based on previous invariant moments the novel proposed approach consists of four stages: 1) image segmentation of vegetation cover and regions definition, 2) labelling of disconnected regions, 3) extraction of the $\mathrm{Hu}$ invariant moments for each region, and 4) discrimination of both monocots and dicots regions by means of the SFI.

The segmentation of the image is a two-steps process. First, it applies the equation (1) to each pixel of the original image with the aim of isolating the vegetation cover [33].

$$
I S=r \cdot I(R)+g \cdot I(G)+b \cdot I(B)
$$

where $r=-0.884, g=1.262, b=-0.311$. The greyscale image resulting is then binarized by using a threshold that was set to 10 in this case (Fig. 2b).

After that, an opening morphologic operation is applied for enhancing the regions, avoiding the overlapping among regions belonging to different weed types, and for 
removing in the image the pixels belonging to noise with the minimum alteration of those belonging to monocots and dicots.

The opening operation is accomplished with a structural element that symmetrically operates in all spatial directions, i.e. a $5 \times 5$ matrix known as diamond.

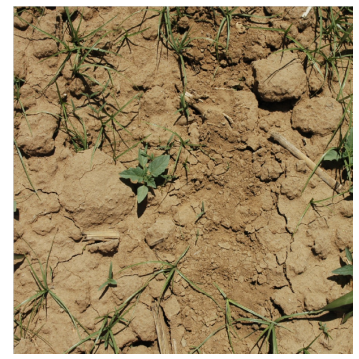

(a)

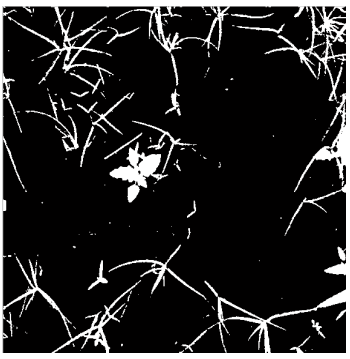

(b)

Fig. 2. (a) Image with mixed monocots and dicots. (b) Segmentation of the vegetation cover of image (a).

In the second stage, the regions are labelled following the procedure described in [34], which basically finds the connected components (regions in this case) in a binary image. In this method, all the pixels in the same region are given the same level. The searching of the connected components is done in top-to-bottom scan order, i.e. all pixels in the first connected component are labelled as 1 , those in the second as 2 and so on.

Once all regions have been labelled, the seven $\mathrm{Hu}$ invariant moments are computed for each region. Therefore, the attributes are the seven mentioned above, i.e. $\Omega \equiv\left\{\phi_{1}, \phi_{2}, \phi_{3}, \phi_{4}, \phi_{5}, \phi_{6}, \phi_{7}\right\}$, where $\phi_{i} \in[0,1]$ and it is associated to the seven $\mathrm{Hu}$ moments.

Then each region is matched with its class of weed. This is a decision that can be made through the combined SFI method, such as it is briefly described below.

The SFI requires the computation of the relevance assigned for each attribute, from which the so-called fuzzy densities can be computed. This is solved by computing the $\lambda$-fuzzy measure [29]. In the proposed approach the calculation starts with selecting a set of seven fuzzy measures, that we will call $g^{1}, g^{2}, g^{3}, g^{4}, g^{5}, g^{6}, g^{7}$ according to [29], each one representing the individual relevance (strength or competence) of the associated attribute in $\Omega$.

The value of $\lambda$ needed for calculating $g^{i}$ is obtained as the unique real root greater than -1 of the polynomial,

$$
\lambda+1=\prod_{i \in \Omega}\left(1+\lambda g^{i}\right), \quad \lambda \neq 0
$$

As it was previously mentioned, SFI is suitable for combining classifiers. Nevertheless, it requires a previous training stage to adjust some parameters. In that approach, SFI learns the relevance for each classifier, so that, during the combination, 
every classifier intervenes with a specific different weight on the final decision. In our combined SFI approach, it is also computed the relevance of each attribute for determining its specific contribution to the decision through the fuzzy densities. As in [35], although with a different criterion, the relevance of each attribute is determined by considering a number of reliable true and false training examples obtained from a set of different regions. The process is as follows: for each region in an image, it is computed the grade of support for its class (monocot or dicot), but considering each one of the seven attributes separately. So, we compute the averaged percentage of error, $p_{1}, \ldots p_{7}$, for the selected regions and for each attribute, based on the expert criterion. Thus, the relevance for an attribute $i$ is computed by (3),

$$
g^{i}=p_{i} / \sum_{j=1}^{7} p_{j}
$$

Once the $g^{1}, \ldots g^{7}$ are obtained and $\lambda$ is found, the SFI works as follows:

1. For a given region, it is obtained a vector as: $\left[\phi_{1}^{\prime}, \phi_{2}^{\prime}, \phi_{3}^{\prime}, \phi_{4}^{\prime}, \phi_{5}^{\prime}, \phi_{6}^{\prime}, \phi_{7}^{\prime}\right]^{T}$; without lost of generality assume that $\phi_{1}^{\prime}$ is the highest value and $\phi_{7}^{\prime}$ the lowest. In this way, this vector is arranged under this criterion, i.e. $\phi_{1}^{\prime}>\phi_{2}^{\prime}>\phi_{3}^{\prime}>\phi_{4}^{\prime}>\phi_{5}^{\prime}>\phi_{6}^{\prime}>\phi_{7}^{\prime}$.

2. Arrange the fuzzy densities correspondingly with the mentioned arrangement, i.e. $g^{l}, g^{2}, g^{3}, g^{4}, g^{5}, g^{6}, g^{7}$ and set the first fuzzy density $g(l)=g^{l}$.

3. For $t=2$ to $7, g(t)$ is calculated recursively by (4),

$$
g(t)=g^{i}+g(t-1)+\lambda g^{i} g(t-1)
$$

4. Calculate for each candidate region $i$, the final degree of support to be matched with each class $l$ as,

$$
\mu_{i}(l)=\max _{h \in \Omega}\left\{\min \left\{\phi_{h}, g(h)\right\}\right\}
$$

5. The decision about the class a region belongs is made by selecting the maximum support $\mu_{i}(l)$ among all classes.

\section{Results}

The images used in this work were taken in maize crops sited in Madrid (Spain) on different days and therefore under varying lighting conditions. A conventional camera positioned atop a tripod was used and the images were acquired in vertical from heights below 1.5 meters, from a space of $50 \times 50 \mathrm{~cm}$ between two maize rows, with an original resolution of $1700 \times 1700$ pixels. Therefore the vegetation that appears in the images is always related to weeds, i.e. monocots, dicots or both kinds of weeds. In 
short, given an RGB image the aim of the developed approach is to estimate in the image the cover percentages of each weed group as well as of soil.

From the sixty-six images available, twenty-eight presented a mixture of weeds. Nineteen images presented only monocots and nineteen only dicots. An important infestation was observed in the $14 \%$ of the images. In this work, fourteen images were selected because represented a wide range of situations. After applying steps 1 (vegetation cover segmentation) and 2 (labelling of disconnected regions) in the selected set of images, one-hundred different regions were extracted and analyzed. In general, the number of regions extracted per image ranged from five to twenty. In the cases where an important infestation was observed, it could be extracted less than five different regions.

Fig. 3 displays, as an example, the regions extracted by the application of steps 1 and 2 over the original image represented in Fig. 2a. Each region appears labelled with a unique label, represented as a colour in a scale for visualization purposes.

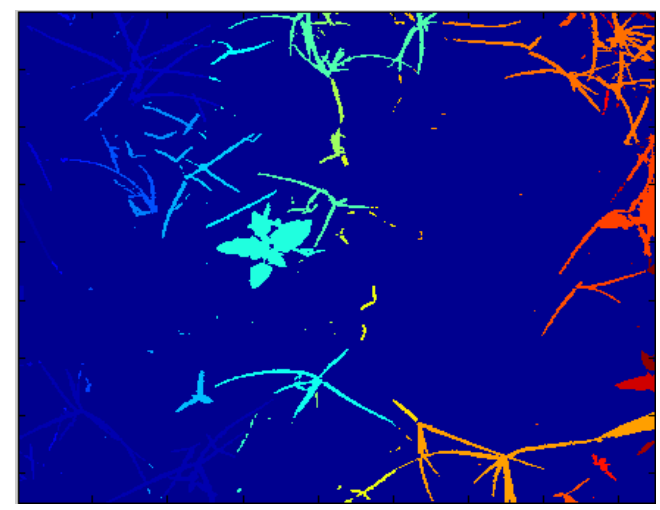

Fig. 3. Labeling regions. Each isolate region appears identified by a unique color.

The tests corresponding to the SFI strategy have been carried out with fourteen images including one-hundred different regions belonging to monocots and dicots. We use four of them (twenty-eight regions) for computing the relevance of each attribute for SFI, from which the fuzzy densities can be obtained. According to the explanation in section 2, the averaged percentage of error, $p_{1}, \ldots p_{7}$, are: $p_{1}=8\left(\phi_{1}\right), p_{2}$ $=10\left(\phi_{2}\right), p_{3}=15\left(\phi_{3}\right), p_{4}=25\left(\phi_{4}\right), p_{5}=21\left(\phi_{5}\right), p_{6}=23\left(\phi_{6}\right)$ and $p_{7}=19\left(\phi_{7}\right)$. Based on (3), the fuzzy values are exactly the following: $\mathrm{g}^{l}=0.066, \mathrm{~g}^{2}=0.083, \mathrm{~g}^{3}=$ $0.124, \mathrm{~g}^{4}=0.207, \mathrm{~g}^{5}=0.173, \mathrm{~g}^{6}=0.190$ and $\mathrm{g}^{7}=0.157$. As one can see, the most relevant attribute is the first one.

At a second stage, we apply the SFI approach region by region for the remainder ten images, as described in section 2.

Furthermore, it is available the information of class membership provided by the expert criterion. Thus, for each region in an image it is known its correct class according to the expert knowledge and this information can be used to compute the percentage of error of the proposed approach. For each one of the seventy-two regions 
obtained from the remainder ten images used for testing, the error for each region and the average of these errors are computed.

According to the $\mathrm{Hu}$ moments extracted for each region, some preliminary conclusions can be obtained for each kind of weed. In monocot weeds, Hu moments tend to have negative values in the fifth, sixth and seventh moment and in the case of moments that give a positive value, they are close to 1 or 2 . In dicot weeds, the values in the seven moments are close to zero and never reach 1 . They may have negative values in the fifth, sixth and seventh moment, but these values are very close to zero. These assertions can be confirmed in Table 1, which shows the Hu invariant moments obtained from fifteen regions of each kind. These regions were randomly extracted from the images used in this work, belonging to the two types of weed under study, Fig. 4.

Table 1. Invariant moments of $\mathrm{Hu}$ obtained from fifteen regions of each kind

\begin{tabular}{|c|c|c|c|c|c|c|c|}
\hline Weed & $\phi_{1}$ & $\phi_{2}$ & $\phi_{3}$ & $\phi_{4}$ & $\phi_{5}$ & $\phi_{6}$ & $\phi_{7}$ \\
\hline \multirow[t]{15}{*}{ Monocot } & 1.7535 & 2.0493 & 2.2928 & 0.8794 & 1.1990 & 1.2571 & -0.3485 \\
\hline & 1.3380 & 1.3438 & 2.0149 & 1.3801 & 2.3014 & 1.5997 & 0.0134 \\
\hline & 1.6944 & 0.9968 & 2.1177 & 0.1024 & 0.0359 & -0.0080 & -0.0314 \\
\hline & 1.6345 & 1.0468 & 2.1471 & 0.4223 & -0.3960 & -0.4321 & 0.0702 \\
\hline & 1.5036 & 0.9277 & 1.0881 & 0.3654 & 0.1670 & 0.2495 & -0.1587 \\
\hline & 1.0571 & 0.3035 & 1.2304 & 0.0342 & -0.0023 & -0.0046 & 0.0066 \\
\hline & 1.2608 & 1.2070 & 0.2663 & 0.2907 & 0.0808 & 0.3193 & -0.0043 \\
\hline & 1.1625 & 0.5494 & 0.7047 & 0.0474 & -0.0016 & -0.0225 & -0.0085 \\
\hline & 1.7169 & 2.2861 & 2.0890 & 0.2256 & -0.1469 & -0.3326 & 0.0492 \\
\hline & 1.4536 & 1.1760 & 2.0314 & 0.4380 & 0.3875 & 0.3890 & -0.1433 \\
\hline & 1.6496 & 2.5818 & 0.6256 & 0.4177 & 0.2127 & 0.6468 & -0.0188 \\
\hline & 1.1978 & 0.3617 & 1.4753 & 0.1781 & -0.0877 & -0.1067 & 0.0253 \\
\hline & 1.6589 & 1.8999 & 2.2096 & 0.7830 & 1.0055 & 0.8419 & -0.2225 \\
\hline & 1.1070 & 0.2534 & 0.5144 & 0.0242 & 0.0013 & 0.0121 & -0.0024 \\
\hline & 1.3074 & 1.0152 & 1.6444 & 0.7993 & 0.8766 & 0.8044 & -0.2670 \\
\hline \multirow[t]{15}{*}{ Dicot } & 0.2982 & 0.0424 & 0.0030 & $247 \mathrm{e}-9$ & $-41 e-12$ & $-29 e-8$ & $-21 \mathrm{e}-10$ \\
\hline & 0.2595 & 0.0189 & 0.0015 & $105 e-9$ & $66 e-12$ & $13 e-10$ & $-42 e-11$ \\
\hline & 0.2605 & 0.0154 & 0.0078 & 0.0004 & $6 e-7$ & $42 e-6$ & $52 \mathrm{e}-9$ \\
\hline & 0.2799 & 0.0071 & 0.0177 & 0.0015 & $73 e-7$ & $12 e-5$ & $16 e-7$ \\
\hline & 0.4753 & 0.1139 & 0.0585 & 0.0108 & 0.0003 & 0.0036 & $-11 e-7$ \\
\hline & 0.2690 & 0.0382 & 0.0004 & 0.0002 & $43 e-9$ & $27 e-6$ & $69 e-11$ \\
\hline & 0.2343 & 0.0032 & 0.0007 & 0.0001 & $25 e-9$ & $31 e-7$ & $-57 e-10$ \\
\hline & 0.2971 & 0.0019 & 0.0261 & 0.0005 & $19 e-7$ & $99 e-7$ & $-57 e-9$ \\
\hline & 0.1981 & 0.0056 & 0.0002 & $59 e-7$ & $19 e-11$ & $37 e-8$ & $6 e-11$ \\
\hline & 0.1966 & 0.0066 & 0.0009 & $54 e-6$ & $12 \mathrm{e}-9$ & $43 e-7$ & $-3 e-9$ \\
\hline & 0.1926 & 0.0030 & $76 e-5$ & $3 e-6$ & $1 e-10$ & $1 \mathrm{e}-7$ & $1 e-10$ \\
\hline & 0.2094 & 0.0053 & 0.0018 & $63 e-6$ & $-1 e-9$ & $5 e-7$ & $2 e-8$ \\
\hline & 0.2008 & 0.0029 & 0.0019 & $99 e-6$ & $37 e-9$ & $33 e-7$ & $2 e-8$ \\
\hline & 0.2108 & 0.0036 & 0.0041 & $16 e-6$ & $37 e-10$ & $9 e-7$ & $2 e-10$ \\
\hline & 0.1991 & 0.0086 & $58 e-5$ & $3 e-5$ & $26 e-10$ & $1 e-6$ & $3 e-9$ \\
\hline
\end{tabular}

These conclusions allow to justify the relevance of $\mathrm{Hu}$ moments in this proposal to discriminate between monocots and dicots. 


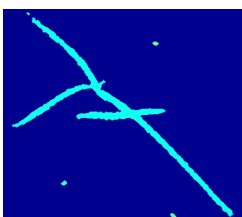

(a)

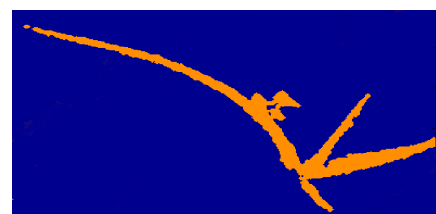

(b)

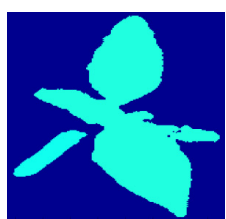

(c)

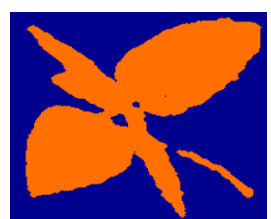

(d)

Fig. 4. (a),(b) Two regions belonging to monocots; (c),(d) two regions belonging to dicots

Through this initial analysis, a classifier based on the Hu moments was designed in order to discriminate between monocots and dicots as described in section 2 . The average percentage of error (compared to the pre-classification done by an expert) obtained with SFI decision making approach is 14.9. The standard deviation $(\sigma)$ obtained is 1.5 . This means that the combination of attributes improve the results. The best individual criterion seems to be the first moment $\left(\phi_{1}\right)$. This implies that it is the most relevant attribute. This agrees with its relevance obtained above, as it has turned out to be the most relevant attribute.

\section{Conclusions}

In this paper we have proposed a strategy for discriminating between monocot and dicot. The method has proven effective and simple; furthermore it is based on colour segmentation, morphological operations and a well known strategy, common operations in image processing.

For each region in an image the seven Hu moments are obtained for determining its correspondence to monocot or dicot. Under the SFI method, the values among seven attributes are combined and a decision for choosing the unique class for each region is made.

The proposed combined strategy works properly when the weeds present an early stage of growth. This is the best moment for applying the herbicide. If the images present states of a higher crop growth, weeds will probably present overlapping and the segmentation process will get difficult mainly due to occlusions that do not allow to see the whole shape of each plant. Nevertheless, the proposed approach provides an useful methodology to discriminate between monocots and dicots in early growth stages.

Although the results achieved can be considered satisfactory, they could be improved by applying Machine Learning techniques to obtain other classifiers able to automate the classification, by means of some induced knowledge which implies the seven moments.

The proposed combined approach can be applied to any environment. In this context, site-specific weed management could mean a significant reduction in herbicide use, which saves the farmer money and benefits the environment. As it was described in the section 1, efficiency is higher if selective treatment is performed for each type of infection instead of using a wide spectrum herbicide. For this reason, this proposal can be essential in the future. 
Acknowledgements. The Spanish Government and the European Union have provided full and continuing support for this research work through projects: PLAN NACIONAL - AGL2011 - 30442 - C02 - 02 (GroW) and UE - CP - IP245986 - 2 (RHEA). The authors wish to acknowledge to the Spanish National Research Council (CSIC) and the European Social Fund (ESF) for the JAE-Doc contract with the first author.

\section{References}

1. Thompson, J.F., Stafford, J.V., Miller, P.C.H.: Potential for automatic weed detection and selective herbicide application. Crop Production 10(4), 254-259 (1991)

2. Marshall, E.J.P.: Field-scale estimates of grass weed populations in arable land. Weed Research 28(3), 191-198 (1988)

3. Johnson, G.A., Mortensen, D.A., Martin, A.R.: A simulation of herbicide use based on weed spatial distribution. Weed Research 35(3), 197-205 (1995)

4. Tian, L., Reid, J.F., Hummel, J.W.: Development of a precision sprayer for site-specific weed management. Transactions of the American Society of Agricultural Engineers 42, 893-900 (1999)

5. Medlin, C.R., Shaw, D.R.: Economic comparison of broadcast and site-specific herbicide applications in nontransgenic and glyphosate-tolerant Glycine max. Weed Science 48(5), 653-661 (2000)

6. Timmermann, C., Gerhards, R., Kühbauch, W.: The economic impact of site-specific weed control. Precision Agriculture 4(3), 249-260 (2003)

7. Tang, L., Tian, L., Steward, B.L.: Classification of broadleaf and grass weeds using Gabor wavelets and an Artificial Neural Network. Transactions of the ASABE 46(4), 1247-1254 (2003)

8. López Granados, F., Jurado-Expósito, M., Atenciano, S., García-Ferrer, A., Sánchez de la Orden, M., García-Torres, L.: Spatial variability of agricultural soils in southern Spain. Plant and Soil 246, 97-105 (2002)

9. Onyango, C.M., Marchant, J.A.: Segmentation of row crop plants from weeds using colour and morphology. Computers and Electronics in Agriculture 39, 141-155 (2003)

10. Ribeiro, A., Fernández-Quintanilla, C., Barroso, J., García-Alegre, M.C.: Development of an image analysis system for estimation of weed. In: Stafford, J.V. (ed.) Proceedings 5th European Conf. On Precision Agriculture (5ECPA), pp. 169-174 (2005)

11. Tellaeche, A., Burgos-Artizzu, X., Pajares, G., Ribeiro, A., Fernández-Quintanilla, C.: A new vision-based approach to differential spraying in precision agriculture. Computers and Electronics in Agriculture 60(2), 144-155 (2008)

12. Tellaeche, A., Burgos-Artizzu, X.P., Pajares, G., Ribeiro, A.: A vision-based method for weeds identification through the Bayesian decision theory. Pattern Recognition 41, 521530 (2008)

13. Burgos-Artizzu, X.P., Ribeiro, A., Tellaeche, A., Pajares, G., Fernández-Quintanilla, C.: Improving weed pressure assessment using digital images from an experience-based reasoning approach. Computers and Electronics in Agriculture 65, 176-185 (2009)

14. Tian, L.F., Slaughter, C.S.: Environmentally adaptive segmentation algorithm for outdoor image segmentation. Computers and Electronics in Agriculture 21, 153-168 (1998) 
15. Brown, R.B., Noble, S.D.: Site-specific weed management: sensing requirements - what do we need to see? Weed Science 53, 252-258 (2005)

16. Lee, W.S., Slaughter, D.C., Giles, D.K.: Robotic weed control system for tomatoes. Precision Agriculture 1(1), 95-113 (1999)

17. Meyer, G.E., Mehta, T., Kocher, M.F., Mortensen, D.A., Samal, A.: Textural imaging and discriminant analysis for distinguishing weeds for spot spraying. Transactions of the ASABE 41(4), 1189-1197 (1998)

18. Ishak, A.J., Hussain, A., Mustafa, M.M.: Weed image classification using Gabor wavelet and gradient field distribution. Computers and Electronics in Agriculture 66, 53-61 (2009)

19. Hemming, J., Rath, T.: Precision agriculture: computer-vision-based weed identification under field conditions using controlled lighting. Journal of Agricultural Engineering Research 78(3), 233-243 (2001)

20. Burgos-Artizzu, X.P., Ribeiro, A., Guijarro, M., Pajares, G.: Real-time image processing for crop/weed discrimination in maize fields. Comput. Electron. Agr. 75, 337-346 (2011)

21. Burks, T.F., Shearer, S.A., Heath, J.R., Donohue, K.D.: Evaluation of Neural-network Classifiers for Weed Species Discrimination. Biosystems Engineering 91(3), 293-304 (2005)

22. Panneton, B., Guillaume, S., Samson, G., Roger, J.: Discrimination of Corn from Monocotyledonous Weeds with Ultraviolet (UV) Induced Fluorescence. Applied Spectroscopy 65(1), 10-19 (2011)

23. Camargo Neto, J., Meyer, G.E.: Crop species identification using machine vision of computer extracted individual leaves. In: Chen, Y.R., Meyer, G.E., Tu, S. (eds.) Optical Sensors and Sensing Systems for Natural Resources and Food Safety and Quality, Proc. SPIE, Bellingham WA, vol. 5996, pp. 64-74 (2005)

24. Sainz-Costa, N., Ribeiro, A., Andujar, D., Dorado, J.: Optimización evolutiva para la construcción de un método de estimación de porcentajes de cobertura de gramíneas y dicotiledóneas. In: Lozano, J.A., Gámez, J.A., Moreno Pérez, J.A. (eds.) Proceedings of the Conference of the Spanish Association for Artificial Intelligence (CAEPIA 2011), vol. 1 (2011)

25. Hu, M.K.: Visual pattern recognition by moment invariants. IRE Trans. Information Theory. 8, 179-187 (1962)

26. Mercimek, M., Gulez, K., Mumcu, T.K.: Real object recognition using moment invariants. Sadhana - Springer India 30(6), 765-775 (2005)

27. Flusser, J., Suk, T., Zitová, B.: Moments and Moment Invariants in Pattern Recognition. John Wiley \& Sons, Ltd. (2009)

28. Herrera, P.J., Pajares, G., Guijarro, M., Ruz, J.J., Cruz, J.M., Montes, F.: A FeaturedBased Strategy for Stereovision Matching in Sensors with Fish-Eye Lenses for Forest Environments. Sensors 9(12), 9468-9492 (2009)

29. Kuncheva, L.: Combining Pattern Classifiers: Methods and Algorithms. Wiley (2004)

30. Klaus, A., Sormann, M., Karner, K.: Segmented-Based Stereo Matching Using Belief Propagation and Self-Adapting Dissimilarity Measure. In: Proc. of 18th Int. Conference on Pattern Recognition, vol. 3, pp. 15-18 (2006)

31. Herrera, P.J., Pajares, G., Guijarro, M., Ruz, J.J., Cruz, J.M.: A Stereovision Matching Strategy for Images Captured with Fish-Eye Lenses in Forest Environments. Sensors 11(2), 1756-1783 (2011)

32. Herrera, P.J., Pajares, G., Guijarro, M., Ruz, J.J., Cruz, J.M.: Segmentation and stereoscopic correspondence in images obtained with omnidirectional projection for forest environments. In: Torreao, J.R.A. (ed.) Stereo Vision, ch. 3, pp. 41-56. In-Tech (2011) 
33. Burgos-Artizzu, X.P., Ribeiro, A., Tellaeche, A., Pajares, G., Fernández-Quintanilla, C.: Analysis of natural images processing for the extraction of agricultural elements. Image Vision Computing 28, 138-149 (2010)

34. Haralick, R.M., Shapiro, L.G.: Computer and Robot Vision, vol. I-II. Addison-Wesley, Reading (1992)

35. Herrera, P.J., Pajares, G., Guijarro, M., Ruz, J.J., De la Cruz, J.M.: Combination of attributes in stereovision matching for fish-eye lenses in forest analysis. In: Blanc-Talon, J., Philips, W., Popescu, D., Scheunders, P. (eds.) ACIVS 2009. LNCS, vol. 5807, pp. 277-287. Springer, Heidelberg (2009) 